\title{
The parallel rod floor test: a measure of ataxia in mice
}

\author{
Helen M Kamens \& John C Crabbe
}

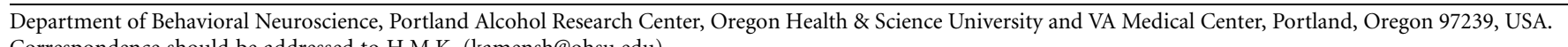
Correspondence should be addressed to H.M.K. (kamensh@ohsu.edu).

Published online 22 February 2007; doi:10.1038/nprot.2007.19

\begin{abstract}
The parallel rod floor test is a new model of ataxia in mice. It allows the simultaneous measurement of ataxia and locomotor activity. This protocol is designed for researchers examining ethanol-induced motor incoordination in mice, but it should be applicable to other sedative/hypnotic drugs and to testing cerebellar mutant mice or mice with engineered genetic defects. This protocol takes $3 \mathrm{~d}$, with the time per day depending on how many animals are tested. The test allows researchers to quantify differences in motor coordination among genotypes of mice that may differ in locomotor activity. Unlike many other methods for assessing incoordination, the parallel rod floor test yields similar patterns of genetic sensitivity across a range of variant forms of the apparatus.
\end{abstract}

\section{INTRODUCTION}

Motor incoordination (or ataxia) has been studied in a number of animal models, both models of neurological disorder and models that provide an index of sensitivity to the sedative effects of drugs of abuse. A number of models have been used to assess ataxia. The most commonly used is the rotarod, where animals are measured to see how long they can continue walking on a rotating $\operatorname{rod}^{1}$. In this task, animals are forced to move to avoid falling from the rod onto a cushioned surface below. Other measures of ataxia also force the animal to move, including the balance beam, which scores hind paw slips from the beam as mice traverse $i^{2}$. Some animals stop moving during this test, forcing the experimenter to encourage movement across the beam by lightly prodding the animal's hindquarter. The grid test ${ }^{3}$, a measure upon which the parallel rod floor test was partially based, also measures foot slips during drug intoxication, and in its original formulation occasionally required that animals be physically encouraged to resume movement. Yet other measures of ataxia allow the animal to explore an arena freely while measures of motor incoordination are taken. One such task is the random hole alley test ${ }^{4,5}$, which demonstrated increased numbers of foot slips through holes in a solid floor in mice with a cerebellar mutation. The measures with the highest apparent validity are observer-rated ataxia scales ${ }^{6}$, recently devised for mice based on earlier scales developed for rats $^{7,8}$. Finally, other tasks measure other physiological responses thought to contribute to ataxia that are independent of locomotor activity. These tests include the screen test ${ }^{9}$, grip strength test ${ }^{10}$ and dowel test ${ }^{11}$.

Although many different models of ataxia exist, it has become apparent that not all are measuring the same underlying behavior. From the list above, an anthropomorphically based classification might conclude that the dowel test, the balance beam test and, to a degree, the various forms of rotarod tests assess the ability to retain balance. The screen test and grip strength test have sometimes been assumed to gauge muscle strength or weakness. From a human perspective, the remaining tasks described above would be thought to reflect relatively straightforward measures of true ambulatory ataxia. Somewhat deeper thought would lead the experimenter to reflect that several other factors might also be playing a role in some of these behavioral assays, including intact visual function, normal proprioceptive feedback, individual differences in anxiety, motivation to explore or move and capacity for motor learning.

All the above mentioned tasks are sensitive to genetic differences (e.g., among inbred mouse strains or between mutants and wild types) $)^{3,4,12-14}$; however, when the genotypic differences in performance on these tasks were compared across eight inbred strains of mice, strain sensitivities showed only modest correlations among tasks ${ }^{15}$. Thus, the tasks were not tapping a single underlying construct, and 'ataxia' is not a simple, monolithic trait insofar as its genetic contributions are concerned. Although strain sensitivities were indeed well correlated for certain pairs and groups of tasks, these clusters of common genetic influence bore no resemblance whatsoever to the anthromorphically assigned characteristics of specific tasks as reflecting 'balance' or 'muscle strength', for example ${ }^{15}$.

Recently, a new test called the paw slip test was developed to examine phencyclidine-induced ataxia in rats ${ }^{16}$. This task examined the number of times a rat's paw slipped through a standard operant chamber floor consisting of a series of parallel metal rods. In 1975, a similar procedure had been developed for mice called the grid test ${ }^{3}$. Mice were tested for ethanol-induced motor incoordination on a 1$\mathrm{cm}$ wire mesh floor, and the number of times their paws slipped through the floor was used as a measure of motor incoordination. One advantage of the grid test is that it takes into account the general locomotor activity of the mouse, which can be a potential confound when comparing ataxic responses across multiple strains of mice. The grid test revealed differences between two inbred strains of mice, C57BL/6 and DBA/2 (which were also highly divergent in the degree to which ethanol stimulated activity), by expressing the ataxic response to ethanol as foot slips per unit distance traveled during the test. Finally, the original grid test also was sensitive to intoxication induced by sodium pentobarbital ${ }^{3}$. We have developed a new mouse model of motor incoordination that combines the positive features of the grid test and the paw slip test, and we call it the parallel rod floor test ${ }^{17}$ (Fig. 1).

When procedures are adapted in a new laboratory, in many cases only a single version of the apparatus and test protocol are used. One advantage of the parallel rod floor test is that it appears to be robust across fairly subtle changes in how the apparatus is configured and used. For example, we systematically tested a number of different rod diameters and inter-rod spacings to determine whether these parameters influenced the pattern of sensitivity to ethanol across eight inbred strains of mice. Almost all configurations yielded interpretable data. An additional strength is that the test showed dose-dependent sensitivity to ethanol across multiple modestly intoxicating doses. A major advantage in the interpretation of drug effects and/or genetic differences is that the main 
dependent variable (foot slips per centimeter traveled in the apparatus) offers an index of incoordination that is corrected for differences in activity. As the number of foot slips is highly correlated with activity, this correction is necessary. (The grid test shares this advantage with the parallel rod floor test.) Overall, when we compared eight inbred strains of mice for ethanol sensitivity across four floor types, our results suggested that the parallel rod floor test is a reliable and robust measure of genetic contributions to ethanol-induced locomotor incoordination because all variants of the test yielded a similar strain sensitivity pattern ${ }^{17}$. This is in contrast to several other measures of ataxia, such as the rotarod, where the parameters of the task (e.g., rate of acceleration of rotation) have been shown to have large effects on the pattern of strain sensitivity. For example, comparing inbred strain data from an accelerating rod with those from the same apparatus rotating at a fixed speed yielded unrelated patterns of strain differences ${ }^{14}$.

One potential limitation to the use of this test is that it has thus far been used to assess only ethanol-induced motor incoordination. Although previous experience with the grid test suggests the new test will have wide applicability across drugs with sedative effects, this remains to be proven. The task also will need to be validated for use with cerebellar mutant mice and genetically engineered genotypes that may have a more extreme ataxia. For example, the random hole alley test was reportedly sensitive to extreme ataxia in staggerer mutant mice ${ }^{5}$, but we were unable to adapt this apparatus to be sensitive to the modestly intoxicating effects of ethanol ${ }^{17}$. Another aspect of this potential limitation is that certain genotypes of mice behave extremely in the parallel rod floor test but not in other tasks that assess ataxia. We found that 129S1/SvImJ inbreds were so extremely sensitive to ethanol in the parallel rod floor test that their data were hardly comparable to those from other strains, even though this inbred strain is not extremely sensitive in many other ataxia tests ${ }^{6,12}$. A final limitation is that this test is likely measuring only one or a few aspects of motor incoordination, and we remind the reader that multiple measures of ataxia are needed to gain understanding of such a complex trait.

The procedures described below assume that multiple apparatuses are constructed and that mice are tested in groups, or squads, to improve throughput. We describe the procedures for testing sensitivity to $2 \mathrm{~g} \mathrm{~kg}^{-1}$ ethanol.

\section{MATERIALS}

\section{REAGENTS}

- Mice (e.g., inbred, outbred, mutant, genetically engineered) ! CAUTION Experimenters must follow guidelines for the care and use of laboratory animals and obtain prior approval for any behavioral testing from their Institutional Animal Care and Use Committee.

- Saline

$\cdot 20 \%$ (vol/vol) ethanol in saline

$\cdot 10 \%$ isopropyl alcohol in water $(\mathrm{vol} / \mathrm{vol})$

EQUIPMENT

- Stainless steel rods $(1.6 \mathrm{~mm}$ in diameter)

- An acrylic frame $\left(22.75 \times 4 \mathrm{~cm}^{2}\right)$

- Four rubber stoppers (size 8 )

- Stainless steel base plate $\left(21 \times 21.5 \mathrm{~cm}^{2}\right)$

- Acrylic box $\left(15 \times 15 \times 20 \mathrm{~cm}^{3}\right)$

- Accuscan monitors (Accusan, Columbus, $\mathrm{OH}$ )

- Wood $\left(5.08 \times 10.16 \times 40 \mathrm{~cm}^{3}\right)$

EQUIPMENT SETUP

Parallel rod floor apparatus A picture of the apparatus can be seen in Figure 1. The parallel rod floor apparatus is assembled as follows. The floor consists of a series of parallel stainless steel rods $1.6 \mathrm{~mm}$ in diameter with an inter-rod spacing of $6 \mathrm{~mm}$ from the edge of one rod to the edge of the next rod. The rods are spaced by drilling through an acrylic frame made from $22.75 \times 4-\mathrm{cm}^{2}$ side panels, through which they extend $2-3 \mathrm{~cm}$ on each side. All rods should be connected outside the acrylic frame by soldering a separate rod perpendicular to the others. The other components are four rubber stoppers (size 8), a stainless steel base plate $\left(21 \times 21.5 \mathrm{~cm}^{2}\right)$ with an acrylic border that is raised $1 \mathrm{~cm}$ above the plate, a clear acrylic box $\left(15 \times 15 \times 20 \mathrm{~cm}^{3}\right)$ with no bottom and a removable lid, Accuscan activity monitors and associated hardware and software for recording locomotor activity, wood $\left(5.08 \times 10.16 \times 40 \mathrm{~cm}^{3}\right)$ and an Apple IIe computer.
The base plate is placed in the center of the activity monitor. Four rubber stoppers are placed under the base plate to raise the plate $2.5 \mathrm{~cm}$ off of the floor of the activity monitor (this allows the attachment of the electrical clip to the base plate). On top of the floor sits the acrylic box into which the animals are placed for testing (Fig. 1). The base plate and the parallel rod floor are connected with electrical clips, one to each component. When the mouse's paw slips through the parallel metal rods and touches the base plate, it completes a circuit and the contact is recorded as an error by an Apple IIE computer. Our apparatus uses a version of the Accuscan monitor that has eight IR beams evenly spaced along each wall of the activity monitor. Standard $5.08 \times 10.16-\mathrm{cm}^{2}$ pieces of wood are used to elevate the activity monitor so that the IR beams cross the apparatus approximately $1.5 \mathrm{~cm}$ above the parallel rod floor. Make sure to place the floor in the center of the activity monitor so that the two beams from the middle of each wall of the locomotor activity chamber split the testing arena equally.

Although we use an Apple IIE to count foot slip errors, any simple circuit completion detection hardware and software can be substituted (e.g., lickometer circuitry for recording licks at a metal drinking spout). To record activity, we used our existing Accuscan monitors and software. These monitors are placed inside sound-attenuating chambers during the test. Any system based on IR beam interruption should work. We see no reason that any system based on videography would not work, provided that the mice to be investigated were willing to stay in the acrylic box with the lid off (or that the videographic system could record paths through the acrylic lid). For our studies of different floor rod diameters and rod spacings, we constructed separate floors with different rod diameters and inter-rod spacings. A standard operant chamber floor may work fine, if available. 


\section{PROCEDURE}

Day 1

1| Move mice to the testing room 45-60 min before testing to acclimate them to the room (turn on the testing apparatus at this point).

2| Set up the parallel rod floor apparatus and activity apparatus to record during a 15-min test (we collect locomotor data during 5 -min periods, and foot slip error data during 1-min periods).

3| Weigh mice from the first squad and place in small individual holding cages no more than 10 min before testing.

4| Prepare injections for the first squad of animals.

5| Inject saline (vehicle) intraperitoneally (volume based on $2 \mathrm{~g} \mathrm{~kg}^{-1}$ ethanol injection) and place mouse immediately into the center of the parallel rod floor apparatus. Start Accuscan monitor, close sound-attenuating chamber and start the error-counting program.

\section{? TROUBLESHOOTING}

6| When the test is complete, remove the mouse from the chamber and place into its home cage. Clean the parallel rod floor and base plate of fecal boli and urine. Wipe well with 10\% isopropyl alcohol.

7| Repeat Steps 3-6 for any remaining mice to be tested that day.

8| Move mice back to the colony room.

Day 2

9| Repeat Steps 1-8.

Day 3

10| Repeat Steps 1-8, as performed on days 1 and 2, except at Step 5 inject $2 \mathrm{~g} \mathrm{~kg}^{-1}$ ethanol (20\% vol/vol) intraperitoneally and place the mouse immediately into the center of the parallel rod floor apparatus.

\section{TIMING}

Steps 1-2: 45-60 min per day for 3 consecutive days

Step 3: $30 \mathrm{~s}$ per animal for 3 consecutive days

Step 4: $30 \mathrm{~s}$ per animal for 3 consecutive days

Step 5: $45 \mathrm{~s}$ per animal for 3 consecutive days

Step 6: 2 min per animal for 3 consecutive days

Step 7: varies depending on number of mice tested per day for 3 consecutive days

Step 8: 5 min for 3 consecutive days

\section{? TROUBLESHOOTING}

Troubleshooting advice can be found in Table 1.

TABLE 1 | Troubleshooting table.

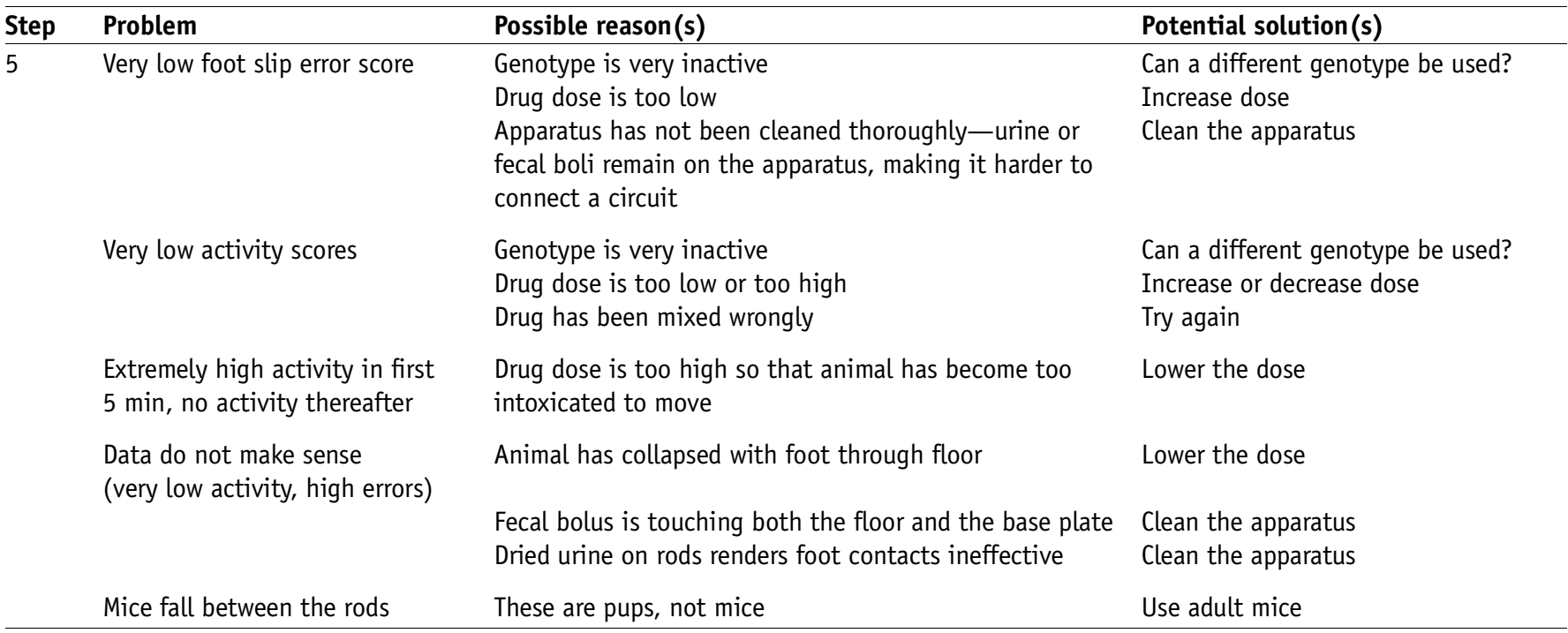




\section{ANTICIPATED RESULTS}

The parallel rod floor test yields two dependent variables: number of errors (foot slips) and horizontal distance traveled $(\mathrm{cm})$. We recommend using a ratio (errors per $\mathrm{cm}$ traveled $\times 100$ ) to index ataxia in this apparatus corrected for individual differences in activity. In a panel of eight inbred strains, we observed ataxia ratios ranging from 7 to 112 across all animals tested, with strain means ranging from 18 to 39 after $2 \mathrm{~g} \mathrm{~kg}^{-1}$ ethanol (individual strain means can be obtained by visiting The Jackson Laboratory's Mouse Phenome Database, http://phenome.jax.org/pub-cgi/phenome/mpdcgi?rtn=docs/home $)^{17}$; mice given higher doses of alcohol or mutants with greater impairment of motor coordination may show higher ataxia ratios.

We used $2 \mathrm{~d}$ of habituation to the effects of saline in this test before testing for drug effects. This is partly for historical reasons and partly because our interest was to index inbred strain differences. In studies of the acute effects of drugs on locomotor activity in our laboratories, we have seen that mouse inbred strains differ greatly in their intrinsic activity. Indeed, strain differences in activity in a novel apparatus are among the most well characterized, and the most stable, in behavioral genetics ${ }^{18}$. We have also seen that with repeated short tests, many strains show pronounced declines in activity and exploration, whereas others maintain a rather high level of activity across repeated tests. We have concluded that the best way to index strain differences in response to ethanol-induced locomotor stimulation or sedation is to habituate the strains to be compared to the apparatus before testing with drug. We have also adopted this practice when exploring other ataxia tests that depend on locomotion (e.g., the grid test). As a result, we adopted a protocol that begins by habituating the animals to the apparatus. It may be that the parallel rod floor test would have given us very similar outcomes if animals had been tested for drug sensitivity without habituation. However, this would have necessitated use of a between-groups design, doubling the cost (the inbred strains we tested cost on average $\$ 26$ per mouse). Although we have adopted a $2 \mathrm{~d}$ habituation procedure, it should be noted that other strains may require more time to habituate to the procedure. Pilot studies may be warranted to determine the optimal number of days required to habituate animals to the test.

Although we used a between-groups design to look at differences among inbred strains to keep cost down, we certainly feel that this protocol could be adapted to experiments benefiting from a within-subject analysis. The eight inbred strains we tested exhibited a low amount of motor incoordination in the absence of ethanol. Other animals, such as those used for neurologic disease models, may exhibit ataxia under basal conditions, and researchers may be interested in pharmacological treatment of such animals. In these cases a within-subjects design may be useful to examine a reduction in motor incoordination. Although such experiments are possible, one must also take into account the effect of multiple tests. In our published data for inbred strains, experiments 3 and 4 included repeated testing of the animals on the same or different floor types. We saw reasonable test-retest reliability in the former case, and the same patterns of strain differences in the latter, which suggests that repeated testing on the parallel rod floor apparatus should be feasible ${ }^{17}$.

We have examined only adult mice in this task. We found no evidence for sex differences in our studies ${ }^{17}$; however, we have no idea whether similar results would be obtained with younger or older mice. For very young mice, the apparatus would clearly need to be rescaled, in terms of both rod spacing and height above the stainless steel plate.

We have not indicated any steps in the PROCEDURE as "Critical." This simply reflects our ignorance about which are actually critical. We believe that all aspects of husbandry, handling and procedural details surrounding the test environment, including the specific experimenter conducting the tests (see ref. 19 for protocol on these topics), potentially have large influences on the outcomes ${ }^{20}$. There is ample evidence to support this belief in the outcomes obtained in mouse behavioral tests ${ }^{21-23}$.

ACKNOWLEDGMENTS Preparation of this protocol was supported by NIH grants AA10760, AA12714, AA015822 and a grant from the US Department of Veterans Affairs.

COMPETING INTERESTS STATEMENT The authors declare that they have no competing financial interests.

Published online at http://www.natureprotocols.com Reprints and permissions information is available online at http://npg.nature.com/ reprintsandpermissions

1. Dunham, N.W. \& Miya, T.S. A note on a simple apparatus for detecting neurological deficit in rats and mice. J. Am. Pharm. Assoc. Am. Pharm. Assoc. (Baltim). 46, 208-209 (1957).

2. Goldowitz, D., Moran, T.H. \& Wetts, R. Mouse chimeras in the study of genetics and structural determinants of behavior. In Techniques for the Genetic Analysis of Brain and Behavior (eds. Goldowitz, D., Wahlsten, D., \& Wimer, R.E.) 271-290 (Elsevier Science Publishers BV, Amsterdam, 1992).

3. Belknap, J.K. The grid test: a measure of alcohol- and barbiturate-induced behavioral impairment in mice. Behav. Res. Methods Instrum. Comput. 7, 66-67 (1975)

4. Guastavino, J-M. A tilted rotational stimulation improves the gait of a cerebellar mutant mouse: the staggerer. Behav. Process 9, 79-84 (1984).
5. Gustavino, J-M., Larsson, K. \& Jaisson, P. Neurological murine mutants as models for single-gene effects on behavior. In Techniques for the Genetic Analysis of Brain and Behavior. (eds. Goldowitz, D., Wahlsten, D. \& Wimer, R.E.) 375-390 (Elsevier Science Publishers BV, Amsterdam, 1992).

6. Metten, P. et al. Observer-rated ataxia: rating scales for assessment of genetic differences in ethanol-induced intoxication in mice. J. Appl. Physiol. 97, 360-368 (2004).

7. Majchrowicz, E. \& Hunt, W.A. Temporal relationship of the induction of tolerance and physical dependence after continuous intoxication with maximum tolerable doses of ethanol in rats. Psychopharmacology (Berl). 50, 102-112 (1976).

8. Sturgeon, R.D., Fessler, R.G. \& Meltzer, H.Y. Behavioral rating scales for assessing phencyclidine-induced locomotor activity, stereotyped behavior and ataxia in rats. Eur. J. Pharmacol. 59, 169-179 (1979).

9. Coughenour, L.L., Mclean, J.R. \& Parker, R.B. A new device for the rapid measurement of impaired motor function in mice. Pharmacol. Biochem. Behav. 6 351-353 (1977).

10. Meyer, O.A., Tilson, H.A., Byrd, W.C. \& Riley, M.T. A method for the routine assessment of fore- and hindlimb grip strength of rats and mice. Neurobehav. Toxicol. 1, 233-236 (1979).

11. Gallaher, E.J., Parsons, L.M. \& Goldstein, D.B. The rapid onset of tolerance to ataxic effects of ethanol in mice. Psychopharmacology (Berl). 78, 67-70 (1982). 
12. Crabbe, J.C. et al. Strain differences in three measures of ethanol intoxication in mice, the screen, dowel and grip strength tests. Genes Brain Behav. 2, 201-213 (2003).

13. Crabbe, J.C. et al. Genotypic differences in ethanol sensitivity in two tests of motor incoordination. J. Appl. Physiol. 95, 1338-1351 (2003).

14. Rustay, N.R., Wahlsten, D. \& Crabbe, J.C. Assessment of genetic susceptibility to ethanol intoxication in mice. Proc. Natl. Acad. Sci. USA 100, 2917-2922 (2003).

15. Crabbe, J.C., Metten, P., Cameron, A.J. \& Whalsten, D. An analysis of the genetics of alcohol intoxication in inbred mice. Neurosci. Biobehav. Rev. 28, 785-802 (2005).

16. Melnick, S.M., Rodriguez, J.S., Bernardi, R.E. \& Ettenberg, A. A simple procedure for assessing ataxia in rats: effects of phencyclidine. Pharmacol. Biochem. Behav. 72, 125-130 (2002)

17. Kamens, H.M., Phillips, T.J., Holstein, S.E. \& Crabbe, J.C. Characterization of the parallel rod floor apparatus to test motor incoordination in mice. Genes Brain Behav. 4, 253-266 (2005).
18. Wahlsten, D., Bachmanov, A., Finn, D.A. \& Crabbe, J.C. Stability of inbred mouse strain differences in behavior and brain size between laboratories and across decades. Proc. Natl. Acad. Sci. USA 103, 16364-16369 (2006).

19. Deacon, R.M. Housing, husbandry and handling of rodents for behavioral experiments. Nat. Protoc. 1, 936-946 (2006).

20. Wahlsten, D. \& Crabbe, J.C. Behavioral testing. In The Mouse in Biomedical Research. Normative Biology, Immunology and Husbandny Vol. 3 (eds. Fox, J.G., Newcomer, C., Smith, A., Barhold, S., Quimby, F. \& Davisson, M.) 514-534 (Elsevier Science Publishers BV, Amersterdam, 2007).

21. Crabbe, J.C., Wahlsten, D. \& Dudek, B.C. Genetics of mouse behavior: interactions with laboratory environment. Science 284, 1670-1672 (1999).

22. Chesler, E.J., Wilson, S.G., Lariviere, W.R., Rodriguez-Zas, S.L. \& Mogil, J.S. Influences of laboratory environment on behavior. Nat. Neurosci. 5, 1101-1102 (2002).

23. Valdar, W. et al. Genetic and environmental effects on complex traits in mice. Genetics 174, 959-984 (2006). 